\title{
A Group Digital Signature Technique for Authentication
}

\author{
Chin-Ming Hsu', Shih-Hsiung Twu', and Hui-Mei Chao'
}

\author{
1 Department of Electronic Engineering \\ Kao-Yuan Institute of Technology \\ Lu-Ju Hsiang 82101, Kao-Hsiung, Taiwan, R.O.C.
}

\author{
2 Department of Electrical Engineering \\ Chung Yuan Christian University \\ Chung-Li 32023, Taiwan, R.O.C.
}

\begin{abstract}
A group digital signature technique using a digital signature algorithm and a challenge-response identification protocol is proposed to provide effective authentication. The proposed digital signature algorithm is based on solving quadratic congruence, factorization, and discrete logarithm problems. Based on the public key infrastructure, group members generate their public-private keys first. The designed authority generates the group member's identity code (ID), the group identity mark, and the group secret key. Every group member keeps his/her private key and the ID for signing. These parameters can ensure only members who can make signatures and provide data authenticity and non-repudiation for any signer. The challenge-response identification protocol with overlapping-shifting-EXOR logical operations is proposed to ensure the signer to obtain group secret key securely and prevent any signer from making false claims. According to the security analysis, the processing time of the proposed approach is faster than the existing RSA and ElGamal group digital signature systems. Moreover, the proposed method would be suited to microprocessor-based devices such as smart cards, computer systems, networks and control systems because of its simplicity, confidentiality, and fast processing speed.
\end{abstract}

Keywords: Group digital signatures, challenge response, authentication, public-private keys, and non-repudiation.

\section{INTRODUCTION}

With the popularity of the Internet and the legislation of digital signatures in Taiwan, transmitting official electronic documentation among different departments to increase the productivity of an institution is encouraged. This encouragement therefore brings group signature authentication problems. Some general properties of group signatures are briefly introduced as follows [1]:

Anonymity:

Given a signature, it is infeasible to find out the identity of the actual signer except the designated group manager or the authority institution.

Un-linkability:

Given two signatures, it is computationally difficult to determine whether the signatures generated by the same member or not.

\section{Unforgeability:}

Only registered members can sign messages on behalf of their group.

Traceability:

In order to identify the actual signer, the group manager or the authority institution is always able to open a valid signature.

Coalition-resistance:

There is no colluding subset of group members can generate a valid signature that the group manager cannot trace.

In the past decade, some group signature techniques have been proposed. Chaum and van Heyst [2] first propose four group signature schemes that are based on solving factorization and discrete logarithm problems. However, two drawbacks exist: (1) the group manager must cooperate with group members in the case of dispute; (2) joining new members or deleting old members needs to change key sizes of the group. Two efficient group signature schemes [3] [4] also are based on solving discrete logarithm mathematical problems. However, they have the same problem: the sizes of secret keys of the group member will increase with the number of signatures. Two efficient group signature techniques for large groups [5-6] are proposed to solve this problem. L. Chen and T. Pedersen [7] propose another information-theoretic group signature scheme. However, this technique allows the group manager to sign messages in the name of any members of group. Moreover, some attacking the existing group signature works are done in [8-11].

In this paper, a new group signature scheme with a challenge-response identification protocol and a digital signature algorithm is proposed to provide effective authentication with three functions: (1) allowing only members to make signatures; (2) remaining actual member who made signatures anonymous; (3) providing 
data authenticity and non-repudiation for any signer in the group. The detail description of the proposed group digital signature scheme is shown in the following section.

\section{PROPOSED GROUP DIGITAL SIGNATURE SCHEME}

The structure of the proposed group digital signature scheme that consists of an authority institution (AI) and group members is illustrated in Figure 1. The interaction between the AI and group members is based on a challenge-response identification scheme. The challenge-response identification scheme supports data confidentiality on transmission and anonymous signature made by the actual member. The Al associated with the challenge-response identification scheme ensures that only members can make the signature, data authenticity, and non-repudiation for any signer in the group.

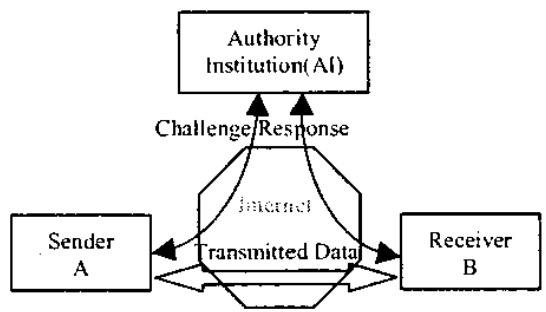

Figure 1. The structure of the proposed group digital signature technique

The digital signature algorithm [12] based on solving quadratic congruence, factorization and discrete logarithm problems is used to correctly identify the signer in the group. The group members first apply this digital signature algorithm to generate their public-private key pairs respectively and register their identities in the Al. According to enrolled group members' identities, the AI then generates a group secret key in the database for further transmission. Five processes are included. They are:

(1) Public-private key generation by group members

(2) Group identity mark and member identity code generation for group members

(3) The challenge-response identification

(4) Signing Process

(5) Verification Process

\section{Public-private key generation for group members}

Step 1: Let $p, q, r$, and $s$ be four prime numbers that satisfy $n_{1}=2 * p^{*} q+1$ and $n_{2}=2^{*} r^{*} s+1$, where $n_{1}$ and $n_{2}$ are also prime numbers. Let $N=n_{1}^{*} n_{2}$, therefore, $\Phi(N)=\left(n_{1}-1\right)\left(n_{2}-1\right)=4^{*} p^{*} q^{*} r^{*} s$, where $\Phi(\mathrm{N})$ is Euler phi-function that is the number of positive integers not exceeding $\mathrm{N}$, which is relatively prime to $N$. If $N$ is prime, $\Phi$ $(\mathrm{N})$ is equal to $\mathrm{N}-1$.

Step2: Choose an odd number $t$ that satisfies following equations:

$$
\begin{aligned}
& X_{1} \equiv t^{2} \bmod (\Phi(N)) \\
& X_{1} * d \equiv 1 \bmod (\Phi(N)) \\
& X_{2} \equiv t^{(i+1)^{2}} \bmod N \equiv t^{K_{1}+2 t+1} \bmod N
\end{aligned}
$$

Step3: Publish the public keys $\left(X_{1}, X_{2}\right.$, and $\left.N\right)$ to the AI. Step4: Keep the private keys ( $t$ and $d$ ) securely.

\section{Group identity mark and member identity code generation for group members}

Let $G$ and $\mathrm{ID}_{i}$ be the group identity mark, ith identity code of a group member, where $i=1,2, \ldots, \mathrm{n}$. These two parameters are set by the AI securely.

\section{The challenge-response identification}

The challenge-response identification process is shown in Figure 2, where $R_{1}$ and $R_{2}$ are random numbers as challenges.

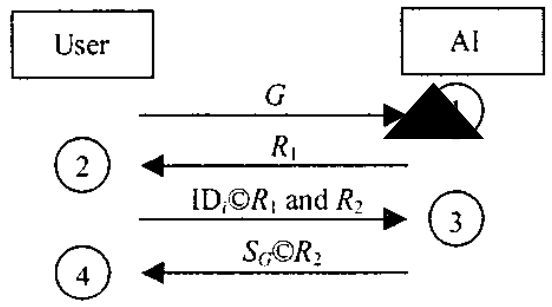

Figure 2. The challenge-response identification scheme

Let $S_{y}$ be the group secret key that is generated by $\mathrm{Al}$ associated with the challenge-response protocol. In this process, the overlapping-shifting with EXOR logical operation symbolized as (c) [13] is used to ensures information transmitted securely. The mathematical expression is described as follows.

$$
\text { Assume } \begin{aligned}
A & =\left[a_{m}, a_{m-1}, a_{m \cdot 2}, \ldots, a_{2}, a_{1}\right] \\
B & =\left[b_{m}, b_{n-1}, b_{n-2}, \ldots, b_{2}, b_{1}\right]
\end{aligned}
$$

Where.

Then, $\quad C=A \oplus B=\left[c_{m}, c_{m-1}, c_{m-2}, \ldots, c_{2}, c_{1}\right]$

$c_{k}=\left\{\begin{array}{l}a_{k} \oplus b_{n-\{m-k)} \oplus b_{n-(m-k+1)} \oplus \cdots \oplus b_{k}, \text { when } n \geq m \\ a_{k} \oplus b_{n} \oplus b_{n-1} \oplus \cdots \oplus b_{n-\{m-k)}, \text { when } n<m, \text { and } k>m-n \\ a_{k} \oplus b_{1} \oplus b_{2} \oplus \cdots \oplus b_{k}, \text { where } n<m, \text { and } k \leq m-n\end{array}\right.$

In order to obtain the $S_{G}$, four steps are included. 
Step 1: The user sends the group identity mark $G$ to the AI

Step 2: The $\mathrm{Al}$ sends the random number $R_{1}$ to the user if the received $G$ is identified correctly.

Step 3: The user sends $I D_{i} C R_{1}$ and the random number $R_{2}$ to the Al. The $\mathrm{AI}$ would take the received $I D_{i} \subseteq R_{1}$ through the decrypting process. If the $R_{1}$ can be decrypted by $\mathrm{ID}_{i}$, the user is then identified.

Step 4: After the user is identified, the AI would response an acknowledgement and send $S_{G} \mathrm{O} R_{2}$ to the user. The user would take the received $S_{G} \mathrm{C} R_{2}$ through the decrypting process to find the group secret key $S_{G}$ for further signing process.

\section{Signing Process}

If $B$ wants to sign a message $m$ to $A$, four steps are included in the signing process.

Step I: B gets the group secret key $S_{G}$ from the AI by the challenge-response protocol.

Step2: B uses his private keys ( $t$ and $d$ ) to sign the message such as

$$
S(m)=\left(m^{d} * t^{d+2 t^{-1}+1} \bmod N\right)
$$

Step 3: B computes two parameters: $X_{S}$ and $P$, by following equations:

$$
\begin{aligned}
X_{S} & =S_{G} \odot \mathrm{ID} \odot S(\mathrm{~m}), \\
P & =G \odot X_{S}
\end{aligned}
$$

Step 4: B sends the $\left(P, X_{S}, S(m), m\right)$ to the A.

\section{Verification Process}

Two steps are included in the verification process.

Step 1: After A receives the signature information $\left(P, X_{S}\right.$, $S(m), m)$ from $\mathrm{B}$, A uses $P$ and $X_{S}$ to verify the received data by calculating $P \odot X_{S}$. If the result is the same as the group identity mark $G$, then the received message is ensured and signed by the group.

Step 2: In the case of dispute, the Al will check the equation $X_{S} \odot S(m)=S_{G} \odot \mathrm{ID}_{i}$. If it is true, the $\mathrm{AI}$ then checks the public key verification table to find the $i^{\text {th }}$ public key to decrypt $S(m)$.

Such as

$$
\begin{gathered}
m^{\prime}=V(S) \equiv S(m)^{x_{1}} * X_{2}^{-1} \bmod N \\
\text { If } \mathrm{m}=\mathrm{m}^{\prime}, \text { the signer is identified. }
\end{gathered}
$$

Proof:

$$
\begin{aligned}
V(S)=m^{\prime} & \equiv X_{2}^{-1} * S^{x_{1}}(\bmod N) \\
& \equiv X_{2}^{+1} *\left(m^{d} * t^{d+2 t^{-1}+1}\right)^{x_{1}} \bmod N \\
& \equiv X_{2}^{-1} * m^{d \cdot X_{1}} * t^{i^{+}+2 t+1} \bmod N \\
& \equiv m * X_{2} * X_{2}^{-1} \bmod N \\
& \equiv m \bmod N
\end{aligned}
$$

\section{Security Analysis:}

The authentication security capability would be provided by the challenge-response identification scheme and the digital signature algorithm. They are analyzed as follows.

(1) The challenge-response identification scheme Using random numbers as challenges and (c) function as the cipher ensures dynamic messages transmitted on the network for each process. These challenges can prevent the user from denying their transmission and any entry from forgery or making false claims. Therefore, the non-repudiation is provided.

(2) The digital signature algorithm

The difficulties of the proposed digital signature algorithm are based on solving three mathematical problems: discrete logarithm, factorization, and quadratic congruence. Therefore, the enough complexity of the proposed method is provided.

\section{IMPLEMENTATION METHODS}

The implementing proposed group signature scheme consists of four procedures: setup, sign, verify, and open. They are described in the following and an example is shown.

Setup

An interactive identification protocol between the Al and the group member is built. Some parameters such as the group identity mark, the user ID, and the user's public-private keys are also set.

\section{Sign}

A signature generating algorithm is applied to generate a signature for a message.

Verify

An algorithm is established to check the validity of a group signature with respect to some related parameters. Open

An algorithm allows the $\mathrm{AI}$ or the group manager to determine the identity of the group member who issued the signature, and provides a proof of this fact.

\section{An example:}

Setup

The interactive challenge-response identification protocol is set as shown in Figure 2. Assuming the group identity mark $G$ is 372 , the user's ID is 965 , user's public keys $\left(X_{1}, X_{2}, N\right)$ equals $(3259,3096,4757)$ and its private keys $(\mathrm{d}, \mathrm{t})$ equals $(2689,113)$.

Sign

Let the group secret key $S_{G}$ be 1453 set by the AI. The user can obtain the $S_{G}$ by the challenge-response 
identification protocol. If the message $m=813$, then the signer's signature can be generated by

$S(m)=m^{d t} * t^{d+2 t^{-1}+1} \bmod N=813^{2089} * 113^{564} \bmod 4757=464$

$X_{S}=S_{G} \odot \operatorname{ID} \odot S(m)=1453$ (c) $965 @ 464=1234$,

$P=G \odot X_{S}=372 @ 1234=251$

Therefore, the related parameters are generated such as:

$\left(P, X_{S}, S(m), m\right)=(251,1234,464,813)$

Verify

By checking $P\left(X_{S}=G\right.$, the validity of a group signature is identified.

Open

By checking Is $X_{S} \odot S(m)$ equal to $S_{\sigma} \odot \mathrm{ID}_{i}$ ? If the answer is yes, the Al then checks the public key verification table to find the $i^{\text {th }}$ public key to decrypt $S(m)$. Such as

$$
\begin{aligned}
& m^{\prime}=V(S) \equiv S(m)^{x_{1}} * X_{2}^{-1} \bmod N \\
& \text { If } \mathrm{m}=\mathrm{m}^{\prime}, \text { the signer is identified. }
\end{aligned}
$$

\section{CONCLUSIONS}

A new group digital signature technique with a challenge-response identification protocol and a digital signature scheme is proposed to 1) allow only members to make signatures, (2) remain actual member who made signatures anonymous, and (3) provide data authenticity and non-repudiation for any signer in the group. The proposed method provides two improvements: faster processing time and the key sizes of the group member will not increase with the number of signatures.

Faster processing time:

Because the parameters of $G, \mathrm{ID}_{i}$, and $S_{G}$ are independent from the group members' signatures, signing, verification, joining new members or deleting old members can be performed by adding or erasing the related public parameters without changing any keys of other members. Therefore, the numbers of calculation are decreased. Furthermore, the proposed method uses only one public-private key pair instead of two pairs for existing works. This reduces the time for generating keys and computing the related parameters.

The sizes of keys of the group member will not increase with the number of signatures:

Because key generation is independent from other group members, therefore, the key sizes of the group member will not increase with the number of signatures. Furthermore, the use of the challenge-response identification protocol and (C) function instead of uses of mathematical calculation to increase the complexity of the scheme, the key sizes are not absolutely needed too long to increase system security.

\section{REFERENCES}

[1] G. M. Liu, Very simple schemes for group signatures, master thesis, Chung Yuan Christian University, June 2003.

[2] D. Chaum and E. van Heyst, "Group signatures," In Andances in Cryptology - EUROCRYPT'91, vol. 547, pp. 257-265, 1991.

[3] L. Chen and T. P. Pedersen, "New group signature schemes," In Advances in Crvptology EUROCRYPT'94, vol. 950, pp.171-181, 1995.

[4] J. Camenisch, "Efficient and generalized group signatures," In Advances in Cryptology EUROCRYPT'97, vol. 1233, pp.465-479, 1997.

[5] W. B. Lee and C. C. Chang, "Efficient group signature based on discrete logarithm," IEE Proc. Comput. Digit. Tech., vol. 145(1), pp. 15-18, 1998.

[6] J. Camenisch and M. Stadler, "Efficient group signature schemes for large groups," In Advances in Cryptology-CRYPTO'97, vol. 1296, pp. 410-424, 1997.

[7] L. Chen and T. Pedersen, "On the efficiency of group signatures providing information theoretic anonymity," In Advances in CryptologyEurocrypt '95, vol. 921, pp.39-49, 1995.

[8] Y. M. Tseng and J.K. Jan, "Improved group signature scheme based on discrete logarithm problem," IEE Proc. Electronic Letters, pp. 1324-1325, 1999.

[9] H. M. Sun, B. J. Chen and T. Hwang. "Cryptanalysis of group signature scheme using self-certified public keys," IEE Proc. Electronic Letters., pp. 1938-1939, 1999.

[10] H. M. Sun, Y. M. Tseng and J. K. Jan, "Improved group signature scheme based on discrete logarithm problem [comment and reply]," IEE Proc. Electronic Letters, pp. 1323-1325, 1999.

[11] Z. Li, Y. Wang, Y. X. Yang and W. Wu, "Cryptanalysis of convertible group signature," IEE Proc. Electronic Letters, pp. 1071-1072, 1999.

[12] J. I. Wang, The study and development of new public-key cryptosystems based on number theory, master thesis, Chung Yuan Christian University, June 1998

[13] C. M. Hsu, The design of a secure and non-repudiation authentication mechanism with data hiding and message encryption, Doctoral dissertation, Chung Yuan Christian University, June 2002. 